1 Universidade do Estado do Rio de Janeiro (Uerj), Instituto de Medicina Social (IMS) - Rio de Janeiro (RJ), Brasil. Orcid: https://orcid org/0000-0002-01351860

helen.nut@gmail.com

2 Universidade do Estado do Rio de Janeiro (Uerj), Instituto de Medicina Social (IMS) - Rio de Janeiro (RJ), Brasil. Orcid: https://orcid org/0000-0001-74816350

cpierantoni@gmail.com

3 Universidade do Estado do Rio de Janeiro (Uerj), Instituto de Medicina Social (IMS) - Rio de Janeiro (RJ), Brasil. Orcid: https://orcid org/0000-0001-87993225

carinne.mag@gmail.com

4 Universidade do Estado do Rio de Janeiro (Uerj), Instituto de Medicina Social (IMS) - Rio de Janeiro (RJ), Brasil. Orcid: https://orcid. org/0000-0002-3262$565 X$

marcia.ney.unesa@gmail. com

5 Universidade do Estado do Rio de Janeiro (Uerj), Instituto de Medicina Social (IMS) - Rio de Janeiro (RJ), Brasil. Orcid: https://orcid org/0000-0001-80823768

rnutricao@gmail.com

\section{A graduação em medicina no Brasil ante os desafios da formação para a Atenção Primária à Saúde}

\author{
Medical undergraduation in Brazil facing the challenges of training \\ for Primary Health Care
}

Swheelen de Paula Vieira', Celia Regina Pierantoni2 ${ }^{\mathbf{2}}$ Carinne Magnago ${ }^{\mathbf{3}}$, Márcia Silveira Ney ${ }^{\mathbf{4}}$, Rômulo Gonçalves de Miranda5.

DOI: 10.1590/0103-11042018S113

RESUMO Este estudo objetivou identificar elementos da formação médica no Brasil, analisando a proximidade deles com os pressupostos da atuação profissional na Atenção Primária à Saúde e das Diretrizes Curriculares Nacionais de 2014. Trata-se de estudo descritivo e exploratório, de abordagem qualiquantitativa, operacionalizado em 2015 e 2016, por entrevistas telefônicas e entrevistas in loco com coordenadores/diretores de cursos de graduação em medicina. Os resultados do survey indicam inclinação para uma formação médica generalista, com ênfase na atenção primária, mas que pouco prepara os alunos para o desenvolvimento de ações multiprofissionais. Nas entrevistas presenciais, foram relatadas fragilidades que limitam o desenvolvimento de competências para atuação na atenção primária, como a resistência e o pouco preparo dos docentes, as condições incipientes das unidades básicas, a rotatividade dos profissionais do serviço e a disputa dos cenários entre instituições de ensino públicas e privadas. Os achados indicam o caminho que está sendo desenhado após a implantação das novas diretrizes de medicina, sugerindo não apenas avanços, mas também desafios que precisam ser superados, especialmente em prol do desenvolvimento de competências para o trabalho colaborativo em equipe.

PALAVRAS-CHAVE Educação médica. Educação de graduação em medicina. Recursos humanos em saúde. Sistema Único de Saúde. Currículo.

ABSTRACT This study aimed to identify elements of medical education in Brazil, analyzing their proximity to the presuppositions of professional performance in Primary Health Care and the National Curricular Guidelines of 2014. It is a descriptive and exploratory study, with a qualitative and quantitative approach, developed in 2015 and 2016, through telephone interviews and on-site interviews with coordinators/directors of undergraduate medicine courses. The results of the survey indicate an inclination towards a generalist medical education, with emphasis on primary care, but that does not prepare students for the development of multiprofessional actions. In face-to-face interviews, weaknesses have been reported that limit the development of competencies for primary care, such as the resistance and lack of preparation of professors, the incipient conditions of basic units, the turnover of service professionals, and the dispute of space 
between public and private educational institutions. The findings indicate the path that is being drawn after the implementation of the new medical guidelines, suggesting not only advances, but also challenges that need to be overcome, especially for the development of skills for collaborative teamwork.

KEYWORDS Education medical. Education, medical, undergraduate. Health Manpower. Unified Health System. Curriculum.

\section{Introdução}

O ensino médico no Brasil vem sendo debatido em diversos fóruns: há um consenso quanto à insatisfação no atendimento das necessidades da população e à necessidade de sua reformulação $0^{1-5}$.

No Brasil, as propostas instituídas no sistema de saúde com a Reforma Sanitária e a implantação do Sistema Único de Saúde (SUS), em especial no que concerne aos cuidados básicos de saúde, não vêm sendo acompanhadas pelos currículos dos cursos de medicina: a educação médica parece ainda não implementar de maneira robusta o alcance de objetivos coerentes com a realidade social, nem elaborar planejamentos eficazes nesse sentido $2, \mathbf{3 , 6 - 8}$.

Apesar das proposições e avanços nos últimos anos, estudos e avaliações do ensino médico contemporâneo mostram que a maioria dos cursos de medicina no País possui o modelo de formação predominantemente fragmentado no ensino das especialidades, seguindo as proposições do Relatório Flexner orientado majoritariamente para atenção hospitalar, com formação mecanicista, biologicista e individualizante, e com necessidade de maior aproximação dos estudantes com as práticas em território, como é o caso da atuação na Atenção Primária à Saúde (APS) 5,7,9,10,11. Entretanto, pode-se vislumbrar um cenário favorável de transformação para construção de novas realidades, com experiências inovadoras e reformas curriculares sendo implementadas em diversas escolas recentemente ${ }^{\mathbf{1 2}}$.

Dessa forma, no Brasil, assim como em outros países a citar exemplos na Europa, com a declaração de Bolonha, em 1999, assim como Estados Unidos e Canadá, o movimento em prol da mudança na formação médica foi ganhando espaço. Movimentos estes que envolvem mudanças nos métodos de ensino, na estrutura curricular, introdução de inovações pedagógicas como 'Aprendizagem baseada por problemas e Medicina Baseada em Evidências', e, também, o ensino com multiplicidade de cenários de prática no campo social ${ }^{13,14}$.

$\mathrm{Na}$ Espanha, por exemplo, a inserção da atenção primária como pauta na graduação em medicina ocorreu apenas em 2008, com a definição de exigências para a verificação de graus universitários oficiais para qualificar o exercício da profissão médica. $\mathrm{O}$ documento contemplou diretrizes que orientaram a estruturação de disciplinas com a finalidade de que o estudante desenvolvesse competências e habilidades para o exercício médico na APS ${ }^{15}$.

No caso da Inglaterra, a formação médica é dividida em três fases: graduação, programa de fundação (Foundation Programme) e especialização (Special Training). Cada uma delas conta com requerimentos de entrada ou exames específicos. A graduação não 
habilita o recém-graduado a exercer a profissão médica, ele confere plenos direitos de seguirem outros caminhos na carreira, como pesquisadores ou acadêmicos, em áreas que não envolvam a assistência direta de pacientes. A função principal da graduação em medicina é fornecer a base necessária para as fases profissionalizantes e remuneradas, por exemplo, o Foundation Programme, no qual os jovens médicos passam dois anos, de modo a auxiliá-los na escolha de suas carreiras profissionais e especialidades ${ }^{\mathbf{1 6}}$.

Historicamente, no Brasil, projetos criados na década de 1980 e 1990, como o Programa de Integração Docente-Assistenciais (IDA), Uma Nova Iniciativa (UNI), Rede Unida e a Comissão Interinstitucional Nacional de Avaliação das Escolas Médicas (Cinaem), representaram iniciativas de transformação na formação de médicos e demais profissionais da saúde, mobilizando discussões principalmente nas universidades,17. Considera-se, contudo, que, nesse primeiro momento, a participação governamental na propulsão dessas iniciativas era ainda elementar; e pouca mudança permanente foi instalada ${ }^{\mathbf{1 8}}$.

Segundo diversos autores, essas iniciativas influenciaram as discussões e mudanças operadas a partir de 2000 , tanto no desenvolvimento de projetos de indução de mudanças na formação em saúde, em particular dos médicos, como na edição das Diretrizes Curriculares Nacionais (DCN) para os cursos de graduação em saúde, a partir de 2001, e a criação do Programa de Incentivos às Mudanças Curriculares dos Cursos de Medicina (Promed), de 2002, em uma articulação dos Ministérios da Saúde (MS) e da Educação (MEC) 2,4,19,20. O Relatório da Organização Mundial de Saúde (2006), publicado em 2006, intitulado 'Trabalhando juntos pela saúde', também foi um marco importante que impulsionou mudanças para a formação de profissionais de saúde no mundo. O documento enfatizava, além dos défices de profissionais de saúde existentes em diversas localidades do mundo e sua irregular distribuição, problemas também inerentes à formação. Nesse documento, mudanças institucionais relacionadas com a prática de ensino, atenção ao conteúdo curricular e aos métodos pedagógicos foram incentivados ${ }^{21}$.

Nesse contexto, destaca-se a criação da Secretaria de Gestão do Trabalho e da Educação na Saúde (SGTES) no âmbito do MS, em 2003, que reforçou a condução das iniciativas de educação na saúde. Inicia-se, nesse período, um segundo movimento de iniciativas e programas indutores de mudanças curriculares, fruto dessa articulação entre o MS e o MEC, agora de forma mais robusta, tais como o Programa Nacional de Reorientação da Formação Profissional em Saúde (Pró-Saúde), o Programa de Educação pelo Trabalho para a Saúde (PET-Saúde) e, mais recentemente, a reedição das DCN da graduação em medicina, em 201422-26.

Assim como nas DCN anteriores, as diretrizes de 2014 defendem que o graduado em Medicina deve ter formação generalista, com capacidade para atuar nos diferentes níveis de atenção à saúde, nos âmbitos individual e coletivo, a partir do desenvolvimento de ações de promoção, prevenção, recuperação e reabilitação da saúde. Ademais, apesar de trazer em seu escopo muitas das definições já tratadas inicialmente, buscaram aprofundar a discussão sobre o conceito de competência enquanto a capacidade de mobilizar conhecimentos, habilidades e atitudes, com utilização dos recursos disponíveis, e exprimindo-se em iniciativas e ações que traduzem desempenhos à prática profissional, em diferentes contextos do trabalho em saúde ${ }^{\mathbf{2 5}, 27}$. Outro diferencial é o detalhamento das três áreas de competências (Atenção à Saúde, Gestão em Saúde e Educação em Saúde), e respectivas subáreas, a serem desenvolvidas durante a formação, o que poderá facilitar a mudança curricular nos mais diversos cursos.

A gestão em saúde, uma das áreas de competências postasnas $\mathrm{DCN}$, requerumcompromisso do aluno com a corresponsabilização 
para a tomada de decisão, o conhecimento e a escolha diagnóstica e terapêutica de acordo com as necessidades e os recursos disponíveis, o compartilhamento de saberes e o trabalho em equipe. Nesse enfoque, a interdisciplinaridade e o trabalho multiprofissional se destacam para atuação nos serviços de APS. Já a educação em saúde requer o compromentimento com a atualidade, a atualização clínica e científica de forma periódica, a gestão do conhecimento, de forma interprofissional, tutorada, compromissada e contextualizada ${ }^{28}$.

Ademais, apesar de manter o internato com duração de dois anos, este deverá ter no mínimo 30\% de sua carga horária total dedicada à APS e à urgência e emergência, com predomínio da APS25.

A atenção à saúde na APS pauta-se nos princípios organizativos do sistema, como a garantia do acesso equitativo aos serviços, a integralidade e resolutividade na assistência e a coordenação na rede de serviços. A inserção dos alunos na comunidade, o vínculo e o cuidado centrado na pessoa, na família e na comunidade são alguns dos pressupostos necessários. Sua aposta para reorientação do sistema público de saúde e consequente expansão reforça a necessidade e, em certa medida, o desafio para a formação de profissionais de saúde aptos a atuar nesse nível da atenção. Não obstante, esse desafio se estende à formação médica, exigindo uma preparação em maior escala de futuros médicos capacitados a atuar em equipe multiprofissional, com uma atuação clínica integral, resolutiva e sensível com relação à diversidade cultural da população ${ }^{\mathbf{7} 29,30}$.

Apesar das tentativas de modificar a fragmentação dos currículos, a desarticulação entre teoria e prática e a ênfase excessiva no componente biológico do processo de adoecer, a partir das inúmeras iniciativas de indução, a formação médica no Brasil ainda parece enfatizar o ambiente hospitalar como lugar por excelência para a aprendizagem prática do estudante. Embora esse cenário esteja se alterando ao longo dos últimos anos, ainda permanecem dúvidas sobre metodologias e estratégias que, de fato, possam reverter esse contexto ${ }^{31}$. Nesse sentido, as novas DCN também parecem tentar cumprir esse papel, uma vez que abordam de maneira mais específica a proposição curricular.

É consensual a incapacidade de adequação das instituições de ensino à velocidade com que são exigidos novos perfis profissionais, tanto no referencial para atuação técnica específica quanto na introdução de concepções pedagógicas que desenvolvam competências para apreensão e aplicação crítica dessas novas técnicas. Tem-se, como exemplo, o crescente envelhecimento da população e o consequente aumento da prevalência de doenças crônicas, que demandam uma APS mais resolutiva a partir do uso de aportes técnicos generalistas, por vezes delegados a profissionais especialistas ${ }^{32}$. Além disso, persiste o paradigma do processo formativo e da regulação do sistema assistencial influenciado por intensa incorporação e especialização tecnológicas, ancorados pela indústria farmacêutica e pelo uso de equipamentos complexos para o diagnóstico e terapêutica, o que interpõe distorções entre as necessidades de saúde da população e a atuação na rede de serviços, bem como das instituições formadoras ${ }^{21,28,32}$.

Pelo exposto, este estudo tem como objetivo central identificar elementos da formação médica no Brasil, analisando a proximidade deles com os pressupostos da atuação profissional na APS e das DCN de 2014.

\section{Métodos}

Trata-se de um estudo descritivo e exploratório, de abordagem qualiquantitativa. Este estudo é um recorte da pesquisa Regulação das profissões em saúde, que investigou a formação de sete profissões de saúde: medicina, enfermagem, odontologia, nutrição, fisioterapia, farmácia e psicologia, coordenada pelo Observatório de Recursos Humanos 
em Saúde do Instituto de Medicina Social da Universidade do Estado do Rio de Janeiro (ObservaRH/IMS/Uerj) em parceria com o Núcleo de Educação em Saúde Coletiva da Universidade Federal de Minas Gerais (Nescon/UFMG). A abordagem quantitativa, de abrangência nacional, foi empregada para a descrição de características do processo de formação em medicina. Para tanto, foi desenvolvido um survey, conduzido com coordenadores/diretores de cursos de graduação em medicina, por meio da técnica de Entrevista Telefônica Assistida por Computador (Etac), entre janeiro de 2015 e junho de 2016.

O universo da Etac correspondeu ao número total de cursos de graduação em medicina existentes em 2013, conforme dados do Instituto Nacional de Estudos e Pesquisas Educacionais Anísio Teixeira (Inep), correspondente a 206. Os gestores de todos esses cursos foram convidados a integrar o estudo, sendo que $78(36,4 \%)$ se recusaram a participar. Ademais, não se obteve retorno de dois cursos, sendo efetuadas mais de 10 tentativas de contato; portanto, a entrevista não foi concluída. Assim, perfazendo um total de 81 perdas, a amostra final correspondeu a 125 cursos participantes da Etac, ou seja, 60,6\% do quantitativo nacional para o ano de 2013 .

Posteriormente, realizou-se uma análise em profundidade por meio de pesquisa in loco, do tipo estudo de casos múltiplos, sendo desenvolvida de agosto de 2015 a setembro de 2016, em parceria com a pesquisa Política, Planejamento e Gestão das Regiões e Redes de Atenção à Saúde no Brasil, coordenada pela Universidade de São Paulo (USP). O recorte se deu a partir das regiões de saúde, sendo 438 destas constituídas no Brasil32. A seleção de regiões de saúde para o estudo baseia-se em uma amostra nacional cujos cenários de estudo foram definidos segundo a tipologia das regiões e outros critérios definidos conforme os objetivos de análise. A tipologia fundamenta-se em indicadores municipais agregados em duas dimensões: situação socioeconômica e oferta e complexidade dos serviços de saúde.
Optou-se por estudar múltiplos casos na tentativa de compreender mais profundamente os fenômenos relacionados com a formação de médicos no Brasil, buscando-se identificar similaridades e disparidades entre cursos de graduação de diferentes localidades, neste caso, regiões de saúde. Neste estudo, as regiões de saúde foram compreendidas como territórios geográficos delimitados e contínuos, estabelecidos por agrupamentos de municípios limítrofes, com características identitárias semelhantes e infraestrutura compartilhada, com a finalidade de integrar os serviços de saúde, conforme apregoa o Decreto $\mathrm{n}^{0} 7.508$, de 28 de junho de $2011^{33}$.

Ao todo, foram selecionadas oito regiões de saúde brasileiras, que somavam 30 cursos de graduação em medicina em 2013. Foram realizados convites prévios aos coordenadores de todos os 30 cursos, por telefone e correio eletrônico, dos quais, nove cursos, de seis regiões de saúde, aceitaram participar mediante disponibilidade no período previsto para a coleta de dados. Considerando que algumas regiões de saúde estudadas possuem apenas um curso de medicina, elas não foram nomeadas a fim de preservar o anonimato dos respondentes; assim, optou-se por identificar os recortes de depoimentos pelo nome da região geográfica onde se localizam os cursos. Nesta amostra, todas as grandes regiões foram contempladas, sendo que, dos nove cursos, três se localizam na região Sudeste.

Os dados da pesquisa de campo foram coletados com auxílio de questionário semiestruturado elaborado, assim como o da Etac, com base nos documentos ministeriais relacionados com a APS (PNAB 2011), na Lei do Execício Profissional da Medicina e nas DCN dos cursos de graduação em medicina de 2001 e de 2014 (atenção à saúde, gestão e promoção) ${ }^{\mathbf{3 4}, 35}$. As entrevistas in loco contemplaram 54 questões relativas ao perfil do entrevistado, caracterização da instituição de ensino e curso, estrutura curricular e perfil de 
competências do egresso. Por ênfase de formação, compreende-se o foco dado pelo curso a conteúdos e práticas que direcionam para a futura atuação profissional em uma área específica.

Para a construção do bloco do perfil de competências do egresso, elaborou-se um rol de ações e procedimentos típicos da APS com base na Portaria $\mathrm{n}^{\circ} 841$, de 02 de maio de 2012, que dispõe sobre a Relação Nacional de Ações e Serviços de Saúde (Renases); na Portaria $\mathrm{n}^{\circ} 2.488$, de 21 de outubro de 2011, que dispõe da Política Nacional de Atenção Básica (PNAB) e nas DCN para os cursos de medicina (2014) $\mathbf{2 5 , 3 5 , 3 6}$. Após selecionar as ações e procedimentos, estes foram agrupados em quatro grupos, para fins de análise, sendo: (i) ações de saúde, (ii) ações gerenciais, (iii) procedimentos técnicos e (iv) procedimentos diagnósticos.

No que tange à organização curricular, verificou-se se havia ou não (i) divisão tradicional entre ciclo básico e profissional, (ii) organização dos conteúdos por meio de disciplinas, módulos integrados ou outra conformação e (iii) atividades integradas com outros cursos de saúde (Educação Interprofissional). Para a análise das áreas de atuação enfatizadas pelos cursos de medicina, optou-se por elencar os três níveis de atenção (Atenção primária, especializada e hospitalar), bem como a gestão e o preparo para docência e pesquisa, com base no que prevê as atuais DCN em seu rol de conhecimentos, habilidades e atitudes requeridas pelo discente ao longo da graduação.

Para a Etac, o referido questionário foi adaptado em formulário eletrônico e reduzido para 32 questões, levando em consideração o meio de aplicação do instrumento e as possibilidades de organização e análise.

Os dados capturados com a Etac foram tratados e analisados por estatística descritiva no Statistical Package for the Social Sciences (SPSS) 22.0. As entrevistas in loco foram gravadas, transcritas e submetidas à análise de conteúdo segundo os passos propostos por Bardin37: (1) pré-análise, etapa na qual os depoimentos já transcritos foram organizados e submetidos à leitura flutuante; (2) exploração do material, em que foram destacados os recortes de ordem semântica do corpus de análise; e (3) inferência e interpretação do material analisado.

Dada a magnitude da pesquisa e a alta densidade dos resultados, ressalta-se que serão apresentados apenas os resultados alinhados ao objetivo estabelecido para este manuscrito a partir de dois tópicos: Pesquisa por Etac: panorama nacional, e Pesquisa in loco: cursos selecionados.

Em atendimento às normas previstas na Resolução $n^{\circ}$ 466/2012, do Conselho Nacional de Saúde, o projeto deste estudo foi submetido e aprovado pelo Comitê de Ética em Pesquisa do Instituto de Medicina Social da Universidade do Estado do Rio de Janeiro, sob Parecer $n^{\circ} 1.248 .858$.

\section{Resultados}

\section{Pesquisa por Etac: panorama nacional}

Dos 125 cursos analisados via Etac, a maior parte é proveniente de Instituições de Ensino Superior (IES) da rede pública $(\mathrm{n}=64 ; 51,2 \%)$, sobretudo de universidades federais $(\mathrm{n}=34 ; 27,2 \%)$. Em conjunto, os cursos de medicina pertencentes à amostra ofertam 10.256 vagas anualmente, com média de 82 vagas por curso, e com percentual médio de preenchimento de $97,2 \%$. Do total de cursos analisados, a maior parte se encontra em municípios com mais de 100 mil habitantes ( $n=65$; $52,0 \%)$ e nas capitais ( $\mathrm{n}=43 ; 34,4 \%)$.

Quanto à distribuição e concentração dos cursos analisados neste estudo via Etac, o panorama segundo as grandes regiões acompanha não apenas a distribuição nacional do total de cursos de 
medicina, mas reflete, também, a distribuição do total de cursos da área da saúde e de todas as áreas, ou seja, maior concentração da oferta na região Sudeste. No caso do presente estudo, $44,4 \%$ dos cursos analisados situam-se nessa região.

Os gestores de curso entrevistados eram, em sua maioria, do sexo masculino ( $\mathrm{n}=72$; $57,6 \%$ ), graduados em medicina ( $\mathrm{n}=116$; $92,8 \%)$ e doutores ( $\mathrm{n}=72 ; 57,6 \%)$. A média de idade do conjunto de gestores foi de 53,4 anos; o tempo médio de formação e de tempo no cargo de gestor de ensino foi de 29,5 anos e de 4,2 anos, nesta ordem.

\section{Organização curricular}

A maior parte dos Projetos Políticos Pedagógicos (PPP) dos cursos analisados foi atualizada em 2015-2016 (54,4\%). Em relação à ênfase de formação do curso, os coordenadores foram inqueridos em relação a algumas áreas de atuação, as quais foram classificadas em uma escala de 1 a 6, em que 1 corresponde à área de menor ênfase e 6, a de maior ênfase. Verificase, na tabela 1, que as áreas mais enfatizadas, na visão dos respondentes, são a APS e a atenção especializada, enquanto as de menor ênfase são a docência e a gestão.

Tabela 1. Ênfases de formação dos cursos de graduação em medicina segundo os gestores entrevistados por Etac. Brasil, 2015-2016 ( $n=125)$

\begin{tabular}{|c|c|c|c|c|c|c|c|c|}
\hline \multirow{3}{*}{ Áreas de atuação } & \multicolumn{8}{|c|}{ Classificação da ênfase de formação sendo 1 a área de menor } \\
\hline & \multicolumn{6}{|c|}{ ênfase e 6 a de maior ênfase ${ }^{\star}(\%)$} & \multirow[t]{2}{*}{ NR (\%) } & \multirow[t]{2}{*}{ Total (\%) } \\
\hline & 1 & 2 & 3 & 4 & 5 & 6 & & \\
\hline $\begin{array}{l}\text { Gestão de serviços de } \\
\text { saúde }\end{array}$ & 24,8 & 26,4 & 24,8 & 10,4 & 8 & 4,8 & 0,8 & 100 \\
\hline Docência & 32,8 & 25,6 & 15,2 & 4,8 & 7,2 & 13,6 & 0,8 & 100 \\
\hline Pesquisa & 12,8 & 24 & 37,6 & 11,2 & 6,4 & 7,2 & 0,8 & 100 \\
\hline Atenção Primária & 2,4 & 0 & 5,6 & 14,4 & 12,8 & 64 & 0,8 & 100 \\
\hline Atenção Especializada & 7,2 & 2,4 & 11,2 & 17,6 & 46,4 & 14,4 & 0,8 & 100 \\
\hline Atenção Hospitalar & 2,4 & 5,6 & 9,6 & 38,4 & 24 & 19,2 & 0,8 & 100 \\
\hline
\end{tabular}

Fonte: Dados das entrevistas telefônicas, 2015-2016

*Classificação em uma escala de 1 a 6, sendo 1 a área de menor ênfase e 6 a de maior ênfase.

Na maioria dos cursos pesquisados, a estrutura curricular é organizada por disciplinas ( $\mathrm{n}=64 ; 51,2 \%)$, porém, já se mostra expressiva a adoção de outras formas de organizações curriculares no universo pesquisado, como a disposição de conteúdos em módulos ( $\mathrm{n}=41 ; 32,8 \%$ ) e eixos temáticos ( $n=13 ; 10,4 \%)$. Verificou-se, ainda, que a Educação Interprofissional (EIP) destacou-se como tendência curricular por meio de atividades integradas e compartilhadas com estudantes de outros cursos, estando previstas em 78 (62,4\%) da matriz curricular dos cursos analisados via Etac.
Em relação à carga horária total, o conjunto de cursos destina maior tempo médio para a oferta de conteúdos teórico-práticos (3.792 horas), seguidos pelos estágios e internatos (3.373 horas) e pelos conteúdos teóricos (3.153 horas).

\section{Cenários de prática}

Em relação aos locais onde os alunos realizam aulas práticas e/ou estágios, foram listadas 12 opções para os entrevistados, que poderiam selecionar quantos campos fossem 
necessários e, ainda, apresentar outros. $\mathrm{Na}$ tabela 2, verifica-se que, entre os cenários mais comumente utilizados, figuram as unidades de APS (100\%), os hospitais gerais $(99,2 \%)$ e as unidades de pronto atendimento $(94,4 \%)$.

\begin{tabular}{lr} 
Tabela 2.Caracterização dos cenários de práticas utilizados nos cursos de medicina analisados. Brasil, 2015-2016 (n=125) \\
\hline Cenários & $\%$ \\
\hline Unidades de atenção primária & 100,0 \\
Hospitais gerais & 99,2 \\
Unidades de pronto atendimento & 94,4 \\
Núcleo de Apoio à Saúde da Família & 91,2 \\
Policlínicas/ambulatórios/clínicas & 88,8 \\
Hospitais especializados & 81,6 \\
Centros de Atenção Psicossocial & 80,8 \\
Lar de idosos & 56,0 \\
Creches e/ou escolas & 52,8 \\
Associação de Pais e Amigos dos Excepcionais ou similares & 48,0 \\
Residências terapêuticas & 40,3 \\
Empresas & 22,4 \\
Outro(s) & 13,6 \\
\hline
\end{tabular}

Fonte: Dados das entrevistas telefônicas, 2015-2016.

No que se refere ao acompanhamento dos discentes durante as práticas/estágios na APS, o mais comum é que eles sejam assistidos pelo próprio corpo docente do curso ( $\mathrm{n}=121 ; 96,8 \%)$ e pelos profissionais da saúde das unidades ( $\mathrm{n}=102 ; 81,6 \%$ ), mas também se verificou que a maioria dos cursos contrata preceptores para este fim $(62,4 \%)$.

\section{Perfil de competências do egresso de medicina}

No intuito de compreender a preparação profissional empiricamente oferecida aos alunos, foram elencados procedimentos e ações típicas da APS. Assim, para cada ação ou procedimento, foi perguntado aos coordenadores se os alunos recebiam preparação para executá-los durante as práticas e estágios da graduação.
As respostas apresentadas na tabela 3 indicam que os cursos analisados nessa amostra fornecem conteúdos necessários para capacitar os alunos para realizar a maioria das ações e procedimentos listados, contudo, na medida em que se avança para ações e procedimentos mais comuns entre algumas especialidades médicas, especialmente oftalmologistas e psiquiatras (como realização de fundoscopia e acompanhamento de pacientes psiquiátricos), e para as práticas e ações mais gerenciais que notadamente exigem trabalho em equipe (como construção de protocolos clínicos e terapêuticos, educação permanente e coordenação de equipes), as proporções diminuem, ou seja, revelando ser competências menos desenvolvidas durante o curso.

Ainda sobre os dados da tabela 3, as médias das respostas indicam maior preparo 
para o conjunto do grupo de procedimentos diagnósticos (89,5\%), ações de saúde (87,4\%) e procedimentos técnicos $(80,9 \%)$, tendo as ações gerenciais a menor média entre os grupos (6,7\%), dado que corrobora aquele que indica a gestão em saúde como uma área pouco enfatizada na formação médica.

Tabela 3. Percentual de entrevistados concordantes, concordantes parciais e discordantes quanto aos alunos serem preparados, durante a graduação em medicina, para desenvolverem ações e procedimentos de saúde selecionados. Brasil, 2015-2016 ( $n=125)$

\begin{tabular}{|c|c|c|c|c|}
\hline Ações e procedimentos & Sim & Parcialmente & Não & NR \\
\hline \multicolumn{5}{|l|}{ Ações de saúde } \\
\hline Atendimento a pacientes com hipertensão e diabetes & 99,2 & 0 & 0 & 0,8 \\
\hline Atendimento à criança (puericultura) & 97,6 & 1,6 & 0 & 0,8 \\
\hline Atendimento ginecológico & 97,6 & 1,6 & 0 & 0,8 \\
\hline Atendimento obstétrico & 96,8 & 2,4 & 0 & 0,8 \\
\hline Tratamento de dermatites & 96 & 3,2 & 0 & 0,8 \\
\hline Tratamento de verminoses & 96 & 2,4 & 0 & 1,6 \\
\hline Atendimento a pacientes com quadro de insuficiência respiratória aguda & 92,8 & 4,8 & 1,6 & 0,8 \\
\hline Tratamento de micoses & 92,8 & 5,6 & 0 & 1,6 \\
\hline Atendimento urgência e emergência & 89,6 & 8,8 & 0,8 & 0,8 \\
\hline Tratar de deficiências nutricionais & 89,6 & 9,6 & 0 & 0,8 \\
\hline Acompanhamento pré-natal de risco & 76 & 16,8 & 6,4 & 0,8 \\
\hline Acompanhamento de pacientes psiquiátricos & 67,2 & 18,4 & 13,6 & 0,8 \\
\hline Atendimento de urgências e emergências psiquiátricas & 44,8 & 31,2 & 21,6 & 2,4 \\
\hline Média & 87,4 & 8,2 & 3,4 & 1,0 \\
\hline \multicolumn{5}{|l|}{ Ações gerenciais } \\
\hline Planejar e realizar ações de promoção da saúde em conjunto com a equipe & 95,2 & 4 & 0 & 0,8 \\
\hline Realizar notificação compulsória & 95,2 & 3,2 & 0 & 1,6 \\
\hline Atestar óbito & 90,4 & 8,8 & 0 & 0,8 \\
\hline Planejar e participar de campanhas de vacinação & 76,8 & 14,4 & 4,8 & 4 \\
\hline Participar da construção de protocolos clínicos & 68 & 18,4 & 9,6 & 4 \\
\hline Coordenar uma equipe multidisciplinar & 65,6 & 27,2 & 4,8 & 2,4 \\
\hline Supervisionar ações individuais e coletivas de profissionais de saúde & 60,8 & 32,8 & 4,8 & 1,6 \\
\hline Participar dos processos de avaliação de qualidade de serviços de saúde & 50,4 & 34,4 & 12 & 3,2 \\
\hline $\begin{array}{l}\text { Organizar processos de educação permanente para os profissionais sob } \\
\text { supervisão }\end{array}$ & 42,4 & 38,4 & 16 & 3,2 \\
\hline $\begin{array}{l}\text { Planejar a distribuição de recursos humanos necessária para a realização } \\
\text { das atividades de saúde }\end{array}$ & 38,4 & 39,2 & 18,4 & 4 \\
\hline Planejar a realização de compra de materiais, insumos e suprimentos & 17,6 & 38,4 & 40 & 4 \\
\hline Média & 63,7 & 23,6 & 10 & 2,7 \\
\hline
\end{tabular}


Tabela 3. (cont.)

\begin{tabular}{|c|c|c|c|c|}
\hline \multicolumn{5}{|l|}{ Procedimentos técnicos } \\
\hline Realizar curativo & 97,6 & 0,8 & 0 & 1,6 \\
\hline Realizar sutura & 96,8 & 0,8 & 0,8 & 1,6 \\
\hline Realizar anestesia local & 94,4 & 0,8 & 3,2 & 1,6 \\
\hline Realizar Papanicolau & 94,4 & 2,4 & 0,8 & 2,4 \\
\hline Realizar incisão e drenagem de abscessos & 92,8 & 4 & 0,8 & 2,4 \\
\hline Realizar otoscopia & 90,4 & 6,4 & 0,8 & 2,4 \\
\hline Realizar remoção de cerume & 80,8 & 5,6 & 10,4 & 3,2 \\
\hline Realizar lavagem gástrica & 80,8 & 8,8 & 5,6 & 4,8 \\
\hline Remover lesões de pele & 77,6 & 12,8 & 7,2 & 2,4 \\
\hline Realizar exame de fundo de olho (fundoscopia) & 76 & 18,4 & 4,8 & 0,8 \\
\hline Remover corpo estranho do ouvido & 66,4 & 16 & 14,4 & 3,2 \\
\hline Realizar teste da orelhinha & 61,6 & 10,4 & 19,2 & 8,8 \\
\hline Remover corpo estranho da córnea ou conjuntiva & 41,6 & 15,2 & 38,4 & 4,8 \\
\hline Média & 80,9 & 7,9 & 8,2 & 3,1 \\
\hline \multicolumn{5}{|l|}{ Procedimentos diagnósticos } \\
\hline Exames laboratoriais & 99,2 & 0,8 & 0 & 0 \\
\hline Raios-X simples & 99,2 & 0 & 0 & 0,8 \\
\hline Eletrocardiograma & 97,6 & 1,6 & 0 & 0,8 \\
\hline Resultado de Papanicolau & 96 & 3,2 & 0 & 0,8 \\
\hline Resultado de teste tuberculínico & 92 & 4 & 0,8 & 3,2 \\
\hline Resultado de ultrassom & 83,2 & 13,6 & 0,8 & 2,4 \\
\hline Resultado de ressonância magnética & 59,2 & 30,4 & 8,8 & 1,6 \\
\hline Média & 89,5 & 7,7 & 1,5 & 1,4 \\
\hline Média do conjunto & 79,9 & 11,9 & 6,2 & 2,1 \\
\hline
\end{tabular}

Fonte: Dados das entrevistas telefônicas, 2015-2016.

\section{Pesquisa in loco: cursos selecionados}

Na pesquisa de campo, primou-se por aprofundar questões que não seriam possíveis na modalidade Etac, como a percepção quanto às novas DCN para a medicina e as possíveis potencialidades e fragilidades relacionadas com o preparo dos alunos para futura atuação na APS.

Dos nove entrevistados nessa etapa, seis eram responsáveis por cursos públicos. Quanto à localização, três são da região Norte, três da Sudeste e um de cada uma das demais grandes regiões do País. Todos os entrevistados eram médicos, dos quais sete homens. A média de idade dos respondentes foi de 51,1 anos, e o tempo médio de formação e de tempo no cargo de gestor de ensino foi de 26,1 e 2,6 anos respectivamente.

\section{Expectativa quanto às novas DCN para a graduação em medicina}

Com relação à atualização do $\mathrm{PPP}$, apenas dois dos nove cursos haviam atualizado os currículos em anos anteriores a 2014 e, 
portanto, antes da promulgação das novas DCN. Sobre isso, foi perguntado aos respondentes a sua percepção sobre o grau de indução das diretrizes para a mudança na formação médica. A maioria afirmou que as diretrizes tendem a provocar poucas mudanças na formação, especialmente por não oferecerem alternativas para superação de desafios relacionados com o quantitativo insuficiente e com a ausência de capacitação docente: "Sinceramente, acho que vai mudar pouco em termos de formação e atuação do médico, pelo menos enquanto não tiver uma política de formação docente" (Região Norte); e ainda: "Nosso corpo docente não está preparado para um processo de formação que contemple metodologias ativas e a autonomia do aluno, a resistência continua" (Região Centro-Oeste). Outra fala que expressa baixa expectativa dos coordenadores no que tange ao poder de indução das novas DCN para a formação médica:

Para as diretrizes funcionarem é preciso ter corpo docente, quantitativo suficiente. A atual relação de docentes-alunos inviabiliza uma melhor inserção dos alunos nos campos de prática. (Região Nordeste).

Um coordenador chama atenção para o descompasso entre o mercado de trabalho em saúde e as exigências de formação.

Na universidade você não consegue formar um profissional para o SUS se o governo não valoriza o SUS. Os profissionais que nós formamos vão todos para o sistema privado porque o governo investe mais no sistema privado do que no SUS. (Região Norte).

\section{A ênfase da formação e o perfil do egresso}

Dos coordenadores entrevistados in loco, $78 \%$ informaram que o atual processo de formação de médicos de seus cursos de graduação enfatiza principalmente a área hospitalar e especializada, em desacordo com os dados capturados pela Etac. Chamou a atenção o descompasso entre a percepção desses coordenadores e a dos coordenadores entrevistados via Etac, já que, nessas entrevistas in loco, a possibilidade de resposta no formato aberto possibilitou que estes comentassem para além das questões pontuadas no Survey via Etac. Assim, entre os motivos citados para a adoção de um modelo de formação de caráter hospitalar estão: a demanda do aluno, que entra no curso com uma especialidade já escolhida, e o despreparo e resistência dos docentes em reordenar a lógica de formação.

O foco dos alunos é especialidade. Via de regra já estão com as especialidades escolhidas. Temos muitas ligas, de neurologia, cardiologia, oftalmologia, que é uma especialização precoce, no nosso entendimento. (Região Centro-Oeste).

Outrossim, para todos os coordenadores entrevistados, apesar de serem contemplados nos PPP, os cenários de prática na APS constituem-se como um dos principais entraves para uma formação mais generalista e para o preparo do aluno para atuação profissional nesse nível de atenção. Dentre as dificuldades, foram destacadas a rotatividade ou ausência de médicos nas equipes de APS e a precária infraestrutura física das unidades.

O problema é a estrutura da atenção básica e, também, a grande rotatividade de médicos. Então, às vezes você programa uma atividade na unidade para a turma e, de repente, o médico sai e a unidade fica sem esse profissional. E aí tem que tentar remanejar para outra unidade. (Região Sudeste).

Outra situação-problema destacada foi a competição por campos de prática entre as instituições públicas e privadas. Segundo os coordenadores, para assegurar esses espaços, as instituições privadas fornecem 
contrapartidas financeiras às unidades e/ ou contratam preceptores específicos para acompanhamento do aluno, estratégias que, via de regra, não podem ser adotadas pelas instituições públicas.

Os cenários são muito disputados pelas instituições privadas, que também são obrigadas a passar por esses campos. Então, elas usam algumas estratégias, como contratar o profissional que está naquele setor para ficar com os alunos. [...] Nós não podemos pagar. (Região Norte).

Apesar dos problemas enfrentados, os respondentes classificam a matriz curricular do curso como boa ou muito boa quanto ao preparo de médicos para atuação na APS: "Eu acho que a matriz é excelente. O formato possibilita a inserção dos alunos na atenção primária desde o início do curso" (Região Sudeste; Região Nordeste).

Quando questionados sobre o perfil dos egressos, os entrevistados o descreveram como sendo, em geral, generalista, tal como apregoam as DCN: "médico generalista, com aptidão para trabalhar no SUS nos três níveis de atenção" (Região Nordeste, Sul e Sudeste). Alguns deles, inclusive, leram o perfil descrito no PPP do curso.

Dois coordenadores, no entanto, assumem que a matriz curricular voltada para a especialização resulta em um profissional com esse perfil.

Os alunos se preparam para especialização em residência, inclusive não se dedicam muito ao internato. [...]. Se eles vão direto para o mercado de trabalho é temporário até passarem na prova de residência, e geralmente é na ESF [...] não gostam e não valorizam, mas vão porque é mais fácil, porque não precisa fazer concurso, você vai e pega um contrato com a prefeitura e começa a trabalhar. (Região Centro-Oeste).

Entre o que a gente quer e o que sai, se tem um meio termo. O nosso aluno ainda sai muito voltado para a especialização, nós ainda temos uma matriz curricular com o viés da especialização, e isso talvez contribua para este perfil. E olha que isso foi modificado com base na última versão do PPP. (Região Norte).

No que diz respeito à organização curricular, a maior parte dos cursos analisados in loco apresenta divisão tradicional por meio de disciplinas isoladas $(\mathrm{n}=7)$. Em dois deles, verifica-se um processo de transição, em que se busca um modelo de ensino mais interprofissional, com indução à vivência multiprofissional com demais cursos da área da saúde. Acerca disso, um dos coordenadores afirmou:

A partir das novas diretrizes temos procurado readequar o modelo de formação. Ainda temos muito o que avançar, especialmente para prepará-los para atuação na Atenção Primária, trabalhar em equipe etc. Temos trabalhado para um modelo curricular menos fragmentado e com mais vivência com outras graduações de saúde. (Região Sudeste)

\section{Discussão}

O fortalecimento de sistemas nacionais de saúde que se baseiam em uma APS robusta passa por questões cruciais, tal como a formação de profissionais de saúde com o preparo adequado para atuar nesse ponto da atenção. A APS, enquanto estratégia adotada para reorientação do SUS e sua consequente expansão, impulsionou para a maior necessidade de formar profissionais aptos para atuar no trabalho em equipe multiprofissional na busca da integralidade do cuidado. Assim, se por um lado um nó crítico para o fortalecimento da APS é a provisão de profissionais (sobretudo médicos) para atuação nas unidades básicas de saúde, por outro, a qualificação e adequação da formação médica para a APS também se faz imperativa7,38-40.

Os resultados do presente estudo trazem 
contribuições para este debate e revelam, no universo estudado, diferenças entre os cursos no que tange aos aspectos curriculares, com ênfases distintas quanto ao foco do ensino; em parte, revelando o processo de modificação em curso nas escolas médicas nos últimos tempos, tal como assinalam alguns estudos,18. Destarte, enquanto os cursos analisados via Etac apresentaram maior ênfase curricular voltada para a APS, verificou-se que os cursos analisados in loco mostraram maior ênfase no nível hospitalar.

Pode-se, contudo, desvelar por meio da pesquisa in loco que entre as fragilidades para maior inserção dos alunos nos campos de prática da APS está a "competição" entre as IES públicas e privadas. Se por um lado a análise da Etac apontou que todos os cursos (esfera pública e privada) se utilizam dos campos da APS para as suas práticas, estando garantido pela readequação dos PPP; por outro, por meio da pesquisa in loco, pôde-se compreender as dificuldades para a inserção dos alunos nesses campos. Isso se deve ao fato de que maior parte das IES privadas não possui serviço próprio de saúde, utilizando-se da rede instalada do SUS, competindo com as IES públicas pela demanda por campos de estágios. Estas últimas, por sua vez, apresentam enorme desvantagem na oferta de contrapartida financeira e estrutural para o serviço em relação às IES privadas.

Estes achados apontam que, apesar da modificação nos PPP com vistas a contemplar a APS esteja sendo realizada com mais efetividade, é necessário concentrar mais esforços para que, na prática, garanta-se a operacionalização desses projetos políticos pedagógicos, haja vista as fragilidades apontadas pelos respondentes in loco.

Ainda sobre os campos de prática, Izecksohn et al.41 destacaram que, além da garantia da inserção no cenário da APS, a existência de docentes e preceptores qualificados e experientes no âmbito da atenção primária é essencial para a construção de uma APS de qualidade, garantindo assim o desenvolvimento de competências adequadas e promovendo a articulação entre a teoria e a prática.

Não é novo o entendimento de que o papel do docente tem sido considerado determinante para o êxito das iniciativas de indução de mudanças curriculares ${ }^{\mathbf{4 2}, 43}$. Assim, resultado deste estudo que também merece destaque é o que aponta o despreparo e a resistência dos docentes como empecilhos para o reordenarmento da lógica de formação médica. Historicamente, os formadores tendem a assumir os mesmos modelos didáticos pelos quais foram formados, implicando um processo de reprodução e legitimidade das práticas pedagógicas mais tradicionais. Intervir nesse processo de naturalização profissional exige, dessa forma, um trabalho sistematizado de reflexão e educação permanente, baseado na desconstrução da experiência 44-46.

Sobre esse aspecto, as diretrizes de 2014 dispõem que o curso de graduação em medicina deverá manter programa permanente de desenvolvimento e valorização docente, a fim de dar sustentabilidade aos processos de mudanças idealizados pelo novo texto das diretrizes $^{\mathbf{2 4 , 4 2}}$. Isto porque é o docente que possibilita pôr em prática o que documentalmente está posto nos projetos pedagógicos do curso, dado que, enquanto mediador do processo ensino-aprendizagem, é dele dependente o emprego de metodologias eficazes para a apresentação dos conteúdos curriculares, que viabilizarão o desenvolvimento das competências profissionais ${ }^{44}$.

Para tanto, não basta apenas o melhor desempenho docente, também é importante que a matriz curricular esteja adequada ao modelo de formação que se quer adotar. Nessa direção, as DCN de 2014 orientam para uma composição curricular plural que desenvolva competências para atenção à saúde, gestão em saúde e educação em saúde, e que contemple a inserção precoce do aluno nos diferentes serviços do SUS, em especial na APS e urgência e emergência, para que vivenciem o trabalho em equipe multiprofissional e lidem com problemas de saúde reais ${ }^{\mathbf{2 4}}$. 
Com o objetivo de garantir uma formação médica alicerçada no sistema nacional de saúde e que resulte em um profissional com perfil capaz de lidar com os problemas de saúde mais prevalentes, inúmeras iniciativas político-pedagógicas vêm sendo adotadas pelo MS. Busca-se, incisivamente, redirecionar o modelo curricular hegemônico dos cursos de graduação em medicina, que fragmenta a formação em dois ciclos (básico e profissional) e tem por objeto central a doença, para um que seja integrador e promotor de saúde ${ }^{11,17,18}$. Acerca dessa fragmentação, o ensino médico ainda mantém uma forte dissociação entre a teoria e a prática, em que o aprendizado é compartimentado, acabando por fragmentar o conhecimento, contribuindo para o surgimento da escolha da especialização de maneira precoce, além de hipervalorizar as partes em detrimento do todo ${ }^{11,18}$.

Vieira $^{47}$, que realizou pesquisa com cursos de medicina do estado do Rio de Janeiro, evidenciou que há heterogeneidade quanto à abordagem de conteúdos referentes à APS na estrutura curricular dos cursos. Outro achado se deve à tendência de descontinuidade dessas disciplinas, que se concentram nos primeiros anos de formação e depois são retomados no internato. Há, então, além de uma lacuna durante a graduação médica, baixa conexão entre os conteúdos teóricos e práticos.

Para Gomes ${ }^{48}$, o modelo, concentrado fortemente no âmbito hospitalar, impõe ao estudante, majoritariamente, o conhecimento da doença, mas não do sujeito e de seu ambiente familiar, social, ambiental e de trabalho.

Campos $^{49}$ aponta que valorizar o ensino da APS é atender a uma demanda social relevante. Argumenta que a APS não se desenvolverá plenamente enquanto não contar com médicos aptos e qualificados para trabalhar em equipe, exercer uma clínica ampliada e participar de atividades coletivas e de gestão. Somando-se a isso, a escolha precoce de uma especialidade médica ainda no âmbito da graduação é geralmente aceita e, em certa medida, estimulada em muitos cursos de medicina. Esse quadro, por sua vez, contribui para o desinteresse dos alunos, especialmente, pelas áreas tidas por eles como 'mais básicas', como é o caso da medicina de família e comunidade ${ }^{50}$. A fragmentação curricular e a ênfase nas especialidades de forma precoce também têm sido apontadas por contribuir para a segmentação do trabalho do futuro médico, colaborando para a menor percepção da globalidade inerente à formação de um perfil de médico generalista em nível de graduação ${ }^{50-52}$.

Assim, o ensino fragmentado em especialidades contribui para um egresso que terá dificuldades em lidar com o cotidiano dos usuários $^{50}$. Sobre isso, este estudo identificou que, apesar da fragmentação curricular caracterizada pela subdivisão em disciplinas ainda ser predominante entre os cursos de medicina analisados, outras modalidades de organização vêm sendo adotadas, a exemplo do currículo modular. Além disso, já se observam tentativas de avançar para modelos pedagógicos que incluam atividades integradas com alunos de outras profissões da saúde, tal como previsto nas DCN de 2014.

Em seu texto, as novas diretrizes apontam orientações voltadas para o desenvolvimento de competências interprofissionais colaborativas e enfatizam que o graduando em medicina deverá aprender interprofissionalmente, a partir da troca de saberes com alunos e profissionais das diversas categorias da área da saúde ${ }^{\mathbf{2 4}, \mathbf{4 4}}$, pressupostos basilares da Educação Interprofissional em Saúde (EIP).

A EIP, definida como "duas ou mais profissões que aprendem com, de e sobre cada uma delas para melhorar a colaboração e a qualidade da assistência"4,53, é assumida globalmente como recurso potente para melhoraria dos sistemas e dos resultados da atenção à saúde ${ }^{54}$. Nessa perspectiva, tem sido adotada em cursos profissionais de diferentes países, como Canadá, EUA, Reino Unido, Austrália e, mais recentemente, Brasil ${ }^{55-58}$.

Estudo realizado por Silva ${ }^{59}$, que analisou a implantação da EIP na Faculdade de Medicina de Marília, identificou a melhor 
aprendizagem para atuação dos futuros médicos em um contexto de trabalho em equipe, tão essencial na APS, como uma das potencialidades dessa estratégia. Nesse tocante, a presente pesquisa demonstrou que os conteúdos relacionados com o trabalho em equipe estão entre aqueles menos oferecidos pelos cursos analisados por Etac. Esse resultado pode ser fruto da própria dificuldade dos docentes em superar a ideia da hegemonia médica, que, por consequência, repassam isso aos alunos.

É preciso, em concomitância com as mudanças que se exigem no campo de formação, estruturação e oferta de serviços de saúde coerentes com o modelo de saúde nacional, o estabelecimento de contratos entre as rede de ensino e saúde que viabilize cenários de prática para todos os estudantes, fortalecendo a integração entre ensino-serviço-comunidade ${ }^{60}$. Não obstante, ainda em nível de proposições para o fortalecimento da abordagem da APS nos cursos de medicina e superação dos desafios apontados, estão: investir, necessariamente, em monitoramento e avaliação do processo de implantação das novas diretrizes curriculares da medicina; promover o processo de educação continuada dos docentes e preceptores para o desenvolvimento das competências requeridas para as mudanças necessárias para $o$ alinhamento de uma formação mais próxima à realidade da APS; ampliar a inserção de profissionais com formação específica em saúde coletiva, atenção primária e medicina de família e comunidade, no corpo docente das IES, além de fortalecer estratégias importantes, como Versus e PET Saúde, voltadas para a formação na atenção primária.

\section{Considerações finais}

Esta pesquisa buscou apresentar um panorama da formação médica do País, localizando os aspectos que se aproximam e/ou se distanciam das DCN de 2014 e das competências requeridas para a atuação profissional na APS.

Cumpre ressaltar que, apesar de a pesquisa não ter alcançado $100 \%$ do universo pretendido, considera-se que o percentual de participantes foi capaz de fornecer dados importantes para a descrição do panorama nacional e das diferenças existentes entre os cursos de medicina quanto à formação, resguardadas as devidas precauções de generalização dos dados. Isto, pois, os dados obtidos nas diferentes etapas foram confrontados entre si e com a literatura científica especializada sobre o tema.

Não se pretendeu traçar comparações entre os cursos estudados nas diferentes etapas, dadas as diferenças amostrais e metodológicas, mas, sim, oferecer informações complementares. A Etac, a partir de uma mostra mais robusta, permitiu-nos caracterizar os currículos dos cursos de medicina do País; o estudo in loco, por sua vez, ajudou-nos a desvelar as fragilidades e as potências no que concerne à operacionalização desses currículos.

Se, de um lado, verifica-se inclinação para uma formação médica generalista, assentada nas disposições das DCN, de outro, apreende-se que as dificuldades relacionadas com o corpo docente e com os cenários práticos podem limitar as mudanças que se desejam alcançar.

De tal sorte, os achados da pesquisa indicam o caminho que está sendo desenhado após a implantação das novas diretrizes curriculares para os cursos de graduação em medicina, sugerindo que muito se avançou, mas que também são muitos os passos que ainda precisam ser dados, em especial em prol do desenvolvimento de competências para o trabalho colaborativo em equipe.

É preciso salientar que, em virtude da pesquisa ter sido realizada logo após a promulgação das novas DCN e do prazo de adequação a elas ainda não ter expirado, novas pesquisas devem ser viabilizadas no sentido de explorar as condutas adotadas pelos cursos que ainda não atualizaram seus PPP, bem como de dar visibilidade para iniciativas de formação inovadoras que possam ser replicadas. 


\section{Referências}

1. Ferreira MA. Educação Médica no Século XXI: O Desafio da Integração da Tecnologia e Humanidades. Rev. Gazeta Médica. 2016; 4(3):156-161.

2. Ciuffo RS, Ribeiro VMB. Brazilian Public Health System and medical training: possible dialog? Interface Comunic. Saúde Educ., 2008; 12(24):125-40.

3. Gomes AP, Rego S. Transformação da Educação Médica: É Possível Formar um Novo Médico a partir de Mudanças no Método de Ensino-Aprendizagem? Revista brasileira de educação médica. 2011; 35(4):557-566.

4. Oliveira NA, Meirelles RMS, Cury GC, et al. Mudanças curriculares no ensino médico brasileiro: um debate crucial no contexto do Promed. Rev. bras. educ. med. Rio de Janeiro. 2008; 32(3):333-346.

5. Ayres JRC, Rios IC, Schraiber LB, et al. Humanidades como disciplina da graduação em Medicina. Rev. Bras. Educ. Med. 2013; 37(3):455-463.

6. Lampert JB. Na transição paradigmática: o que o paradigma da integralidade atende que o paradigma flexneriano deixou de lado. Cadernos da Associação ABEM 2004;1: 23-25.

7. Almeida-Filho N, Lopes AA, Santana LAA, et al. Formação Médica na UFSB: II. O Desafio da Profissionalização no Regime de Ciclos. Revista brasileira de educação médica. 2015; (39):123-134.

8. Koifman L. O modelo biomédico e a reformulação do currículo médico da Universidade Federal Fluminense. Hist. Cienc. Saude-Manguinhos. 2001; 8(1):49-69.

9. Rios IC, Lopes Junior A, Kaufman A, et al. A Integração das Disciplinas de Humanidades Médicas na Faculdade de Medicina da USP - Um Caminho para o Ensino. Rev. Bras. Educ. Med. 2008; 32(1):112-121.

10. Almeida-Filho N. Higher Education and Health Care in Brasil. The Lancet. 2011; 377/9781:1898-1900.
11. Paim J, Travassos C, Almeida C, et al. The Brazilian health system: history, advances, and challenges. Lancet [online]. 2011 [acesso em 2018 jun 2]; (11):60054-8. Disponível em: https://www.thelancet.com/journals/lancet/article/PIIS01406736(11)60054-8/fulltext?code=lancet-site.

12. Nogueira MI. As mudanças na educação médica em perspectiva: reflexões sobre a emergência de um novo estilo de pensamento. Rev. Bras. Educ. Med. 2009; 33(2): 262-270.

13. Santos JLF, Westphal M. Práticas emergentes de um novo paradigma de saúde: o papel da universidade. Estud. av. 1999 [acesso em 2018 ago 19]; 13(35):71-88. Disponível em: http://www.scielo.br/ scielo.php?script=sci_arttext\&pid=S010340141999 000100007\&lng=en $\& \mathrm{nrm}=$ iso.

14. Gonçalves G. Ensino da saúde pública baseada na evidência, Acta Med Port. 2011; 24(2):467-478.

15. Campos JJB, Foster AC, Freire Filho RJ. Lições aprendidas na comparação dos sistemas de saúde brasileiro e espanhol. Espaço para Saúde. 2016; 17(1):121-129.

16. Norman, AH. A formação em medicina de família no Brasil: a necessidade de caminhos convergentes. Revista Brasileira de Medicina de Família e Comunidade. 2014; 9(30):1-2.

17. Teixeira CFS, Coelho MTD, Rocha MND. Bacharelado interdisciplinar: uma proposta inovadora na educação superior em saúde no Brasil. Ciênc Saúde Colet. 2013; 18(6):1635-1646.

18. Batista CB. Movimentos de reorientação da formação em saúde e as iniciativas ministeriais para as universidades. Barbaroi [internet]. 2013 [acesso em 2018 jun 2]; (38):97-125. Disponível em: http://pepsic.bvsalud.org/scielo.php?script=sci_arttext\&pid=S0104$-65782013000100007 \& \operatorname{lng}=p t \& t \operatorname{lng}=p t$.

19. Gonzaléz AD, Almeida MJ. Movimentos de mudan- 
ça na formação em saúde: da medicina comunitária às diretrizes curriculares. Physis Revista de Saúde Coletiva. Rio de Janeiro. 2010; 20(2):551-570.

20. Brasil. Ministério da Saúde. Portaria Interministerial $n^{\circ} 610$, de 26 de março de 2002. Institui o Programa Nacional de Incentivos às Mudanças Curriculares para as Escolas Médicas (Promed). Diário Oficial da União. 1 Abr 2002.

21. Organização Mundial De Saúde - OMS. Trabalhando juntos pela saúde: relatório mundial de saúde 2006. Brasília, DF: Ministério da Saúde, 2007. (Série B, Textos Básicos de Saúde).

22. Oliveira NA. Ensino médico no Brasil: desafios e prioridades, no contexto do SUS: um estudo a partir de seis estados brasileiros [tese]. Rio de Janeiro: Fundação Oswaldo Cruz; 2007. p. 201

23. Pierantoni CR, França T, Magnago C, et al. Graduações em Saúde no Brasil: 2000-2010. Rio de Janeiro: CEPESC; 2012.

24. Brasil. Ministério da Saúde. Ministério da Educação. Programa Nacional de Reorientação da Formação Profissional em Saúde - Pró-Saúde: objetivos, implementação e desenvolvimento potencial. Ministério da Saúde, Ministério da Educação. Brasília, DF: Ministério da Saúde; 2007.

25. Brasil. Ministério da Educação. Conselho Nacional de Educação. Câmara de Educação Superior. Resolução $n^{\circ}$ 3, de 20 de junho de 2014. Institui as Diretrizes Curriculares Nacionais do Curso de Graduação em Medicina e dá outras providências. Diário Oficial da União. 23 Jun 2014.

26. Brasil. Lei ${ }^{\circ} 12.871$, de 22 de outubro de 2013. Institui o Programa Mais Médicos, altera as Leis $\mathrm{n}^{\mathrm{o}}$ 8.745, de 9 de dezembro de 1993, e $\mathrm{n}^{0}$ 6.932, de 7 de julho de 1981, e dá outras providências. Diário Oficial da União. 23 Out 2013.

27. Brasil. Ministério da Educação. Câmara de Educação Superior. Conselho Nacional de Saúde. Resolução CNE/CES/MEC no 4, de 7 de novembro de
2001. Institui as Diretrizes Curriculares Nacionais do Curso de Graduação em Medicina. Diário Oficial da União. 28 Set 2001.

28. Savassi LCM, Dias EC, Gontijo ED. Formação médica, Atenção Primária e interdisciplinaridade: relato de experiência sobre articulações necessárias. Revista Docência do Ensino Superior. 2018; 8(1):189204.

29. Frenk J, Chen L, Bhutta ZA, et al. Health professionals for a new century: transforming education to strengthen health systems in an interdependent world. Lancet. 2010;376:1923-1958.

30. Batista CB, Gonçalves L. Marcos sobre a integração ensino-serviço na formação de profissionais para a saúde. In: Kind L, Batista CB, Gonçalves L, organizadores. Universidade e Serviços de Saúde: Interfaces, desafios e possibilidades na formação profissional em saúde. Belo Horizonte: Ed. PUC Minas, 2011.

31. Gomes AP, Costa JRB, Junqueira TS, et al. Atenção primária à saúde e formação médica: entre episteme e práxis. Rev. Bras. Educ. Med. [internet]. 2012 [acesso em 2018 jun 2]; 36(4):541-9. Disponível em: https://dx.doi.org/10.1590/S010055022012000600014 .

32. Pierantoni CR, Viana ALA, Tavares RAW, et al. Avaliação do curso de atualização em gestão municipal na área de saúde: uma proposta de metodologia. In: Falcão A, Santos Neto PM, Costa PS, Belisário SA orgs. Observatório de Recursos Humanos em Saúde no Brasil: Estudos e Análises. Rio de Janeiro: Fiocruz; 2003.

33. Brasil. Decreto $\mathrm{n}^{0} 7.508$, de 28 de junho de 2011. Regulamenta a Lei no 8.080, de 19 de setembro de 1990, para dispor sobre a organização do Sistema Único de Saúde - SUS, o planejamento da saúde, a assistência à saúde e a articulação interfederativa, e dá outras providências. Diário Oficial da União. 29 Jun 2011.

34. Brasil. Lei $\mathrm{n}^{0} 12.842$, de 10 de julho de 2013. Dispõe sobre o exercício da Medicina. Diário Oficial da 
União. 11 Jul 2013.

35. Brasil. Portaria GM/MS no 2.488 , de 21 de outubro de 2011. Aprova a Política Nacional de Atenção Básica. Diário Oficial da União. 22 Out 2011.SAÚDE DEBATE | RIO DE JANEIRO, V. 42, NÚMERO ESPECIAL 1, P. INICIAL-140, SETEMBRO 2018 A graduação em medicina no Brasil ante os desafios da formação para a Atenção Primária à Saúde 139

36. Brasil. Ministério da Saúde. Renases- Relação Nacional de Ações e Serviços de Saúde. Renases. Brasília, DF: Ministério da Saúde; 2012b. [acesso em 2018 jun 28]. Disponível em: http://conitec.gov.br/images/Protocolos/Renases2012.pdf.

37. Bardin L. Análise de Conteúdo. São Paulo: Edições 70; 2011.

38. Thumé E, Accioli S, Fehn A et al. A formação de recursos humanos para o SUS avanços, desafios e estratégias. [Texto preparatório - Abrascão]. 2018. [acesso em 2018 jun 10]. Disponível em: http:// rededepesquisaaps.org.br/wp-content/uploads/2018/03/Thume\%CC\%81Acioliet-alAFormacao epraticame\%CC\%81dicoseenfermeiros2018.pdf.

39. Giovanella L, Romero RV, Silva HT, et al. Atención primaria de salud en Suramérica: Reorientación hacia el cuidado integral? In: Giovanella L, organizadora. Atención Primaria de Salud en Suramérica. ISAGS: Unasur; 2015.

40. Anands BT. Health workers at the core of the health system: framework and research issues. Health Policy; 2012;105(2):185-191.

41. Izecksohn MMV, Teixeira Junior JE, Stelet BP, et al. Preceptoria em Medicina de Família e Comunidade: desafios e realizações em uma Atenção Primária à Saúde em construção. Ciênc Saúde Colet. 2017;22(3):737-746.

42. Freire Filho JR, Viana CM, Forster AC, et al. New national curricula guidelines that support the use of interprofessional education in the Brazilian context: an analysis of key documents. J. Inter- prof. Care [internet]. 2017 [acesso em 2018 jun 2]; 31(6):754-60. Disponível em: http://www.tandfonline.com/doi/full/10.1080/13561820.2017.1346592

43. Mesquita SKC, Meneses RMV, Ramos DKR. Metodologias ativas de ensino/aprendizagem: dificuldades de docentes de um curso de enfermagem. Trab. Educ. Saúde. 2016; 14(2):473-86.

44. Magnago C, Pierantoni CR, Carvalho CL, et al. Nurse training in health in different regions in Brazil. Rev. Bras. Saude Mater. Infant. [internet]. 2017 [acesso em 2018 jun 8]; 17(Supl 1):219-28. Disponível em: http://dx.doi.org/10.1590/1806-9304201700s100010.

45. Alves CRL, Belisário SA, Lemos JMC, et al. Mudanças curriculares: principais dificuldades na Implementação do PROMED. Rev. Bras. Educ. Med. [internet] 2013 [acesso em 2018 jun 8]; 37(2):157-66. Disponível em: http://www.scielo.br/scielo.php?script=sci_arttext\&pid=S0100$-55022013000200002 \& \operatorname{lng}=$ en\&tlng $=$ pt

46. Vieira JE, Tamousauskas MRG. Avaliação das resistências de docentes a propostas de renovações em currículos de graduação em medicina. Rev. Bras. Educ. Med. [internet]. 2013 [acesso em 2018 jun. 8]; 37(1):32-8. Disponível em: http://dx.doi. org/10.1590/S0104-40602011000100011.

47. Vieria SP. Abordagem da Atenção Básica nos cursos de graduação em medicina: o panorama do estado do Rio de Janeiro [tese]. Rio de Janeiro: Universidade do Estado do Rio de Janeiro; 2015. 163p.

48. Campos GWS. Papel da rede de atenção básica em saúde na formação médica: Diretrizes. 2005. No prelo.

49. Gomes AP, Costa JRB, Junqueira TS, et al. Atenção primária à saúde e formação médica: entre episte e práxis. Rev. bras educ. med. 2012; 36(4):541-549.

50. Cabral Filho WR, Ribeiro VMB. A escolha precoce da especialidade pelo estudante de medicina: um desafio para a educação médica. Rev. bras educ. 
med. 2004; 28(2):133-144.

51. Bittencourt-Costa JR, Romano VF, Costa RR, et al. Active teaching-learning methodologies: medical students views of problem-based learning. Rev. bras educ. med. 2011; 35(1):13-19.

52. Barr H, Hammick M, Freeth D, et al. Evaluating interprofessional education: a UK review for health and social care. London: BERA/CAIPE; 2000.

53. Institute of Medicine. Interprofessional education for collaboration: learning how to improve health from interprofessional models across the continuum of education to practice. Washington (DC): The National Academies; 2013.

54. Barr H, Koppel I, Reeves S, et al. Effective interprofessional education: argument, assumption and evidence. Oxford: Blackwell; 2005.

55. McNair R, Stone N, Sims J, et al. Australian evidence for interprofessional education contributing to effective teamwork preparation and interest in rural practice. J. Interprof. Care [internet]. 2005 [acesso em 2018 jun 6]; 19(6):579-94. Disponível em: http://www.tandfonline.com/doi/full/10.1080/135 61820500412452 .

56. Reeves S, Lewin S, Espin S, et al. Interprofessional teamwork for health and social care. Oxford: Wiley-Blackwell; 2010.
57. Frutuoso MFP, Junqueira V, Capozzolo AA. A experiência de formação (em) comum de nutricionistas na Unifesp, campus Baixada Santista. Saúde debate [internet]. 2017 [acesso em 2018 jun 9]; 41(112):298310. Disponível em: http://dx.doi.org/10.1590/01031104201711224 .

58. Silva RHA. Educação interprofissional na graduação em saúde: aspectos avaliativos da implantação na Faculdade de Medicina de Marília (Famema). Educar em Revista [internet]. 2011 [acesso em 2018 jun 8]; (39):159-75. Disponível em: https://dx.doi. org/10.1590/S0104-40602011000100011.

59. Baldoino AS, Veras RM. Analysis of Service-learning activities adopted in health courses of Federal University of Bahia. Rev. Esc. Enferm. USP [internet]. 2016 [acesso em 2018 jun 10];50(spe):17-24. Disponível em: http://dx.doi.org/10.1590/S0080623420160000300003 .

60. Costa, JRB, Romano VF, Costa RR, et al. A transformação curricular e a escolha da especialidade médica. Rev. bras. educ. med. 2014; 38(1):47-58.

\footnotetext{
Recebido em 11/06/ 2018

Aprovado em 23/08/2018

Conflito de interesses: inexistente

Suporte financeiro: Organização Pan-Americana da Saúde

(Opas)/Ministério da Saúde (MS)
} 